\title{
LARM bot humanoid design towards a prototype
}

Keywords: humanoid robots, design, prototypes

\section{Introduction}

Humanoid designs are elaborated at different levels with several solutions worldwide and many prototypes are available in laboratory frames for further researches and designs as well as few others are on the market for sales in some applications. Challenges are organized (the most famous one is the USA DARPA program) with the aim to stimulate new designs and improvements in challenges for more and more complicate tasks. Those tasks require multi-disciplinary designs in which the mechanical design still pay an important role both for the operation performance and cost features. The LARMbot humanoid design can be understood as the ultimate design of a mechanism based approach from a decennial activity at LARM in designing robotic structures with inspiration or replication of human bodies as an integration of those results with the common aspect in low-cost user-oriented design and operation, ${ }^{1}$ Here the status of the design is presented with the built prototypes of the parts and an attempt of a full humanoid assembly.

\section{The LARM bot humanoid design}

The LARMbot design is a mechanism-based structure that is inspired by the muscular-skeleton structure of the human anatomy considering the bones as per the serial chains and the muscular systems
Volume I Issue 2 - 2017

\author{
Marco Ceccarelli, Daniele Cafolla, Matteo \\ Russo, Giuseppe Carbone \\ LARM: laboratory of Robotics and Mechatronics, University of \\ Cassino and South Latium, Italy
}

\begin{abstract}
Correspondence: Marco Ceccarelli, LARM: laboratory of Robotics and Mechatronics, University of Cassino and South
\end{abstract} Latium, Italy, Email ceccarelli@unicas.it

Received: August 24, 2017| Published:September 07, 2017

per the driving parallel architectures. ${ }^{2}$ In Figure 1 the main conceptual designs are shown as referring to the trunk structure as a combination of serial spine-like chain and a cable parallel manipulator, (Figure 1A) $)^{3,4}$ leg system as with two tripod linear actuated mechanisms, (Figure $1 \mathrm{~B})^{5}$ and the full assembly as an integrated system, (Figure 1C). ${ }^{6}$ The mechanism designs have been protected by patent submissions. The main characteristics can be recognized in a reduced number of actuators also for an efficient operation with high performance in motion and payload, a compact light mechanical design, and a fairly easy manufacturing of the parts (via 3D printing) with commercial components, thanks to the mechanisms designs.

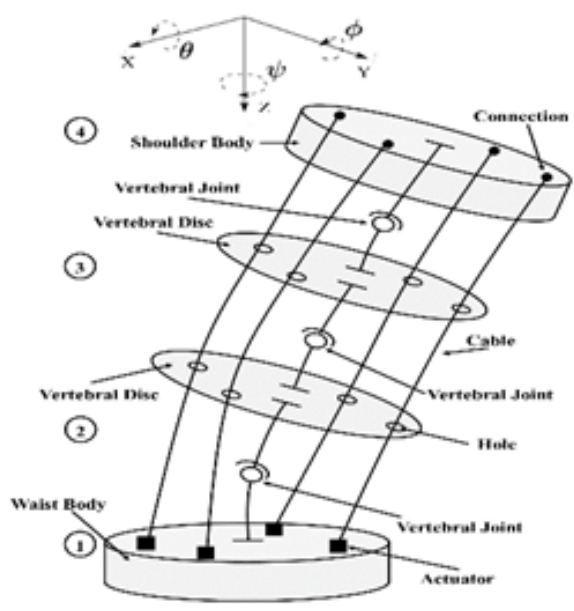

A

Figure I Mechanism design of the LARMbot Humanoid. (A) Torso: The LARM bot prototypes

The design activity has been combined with prototype constructions both to validate the design concepts and to conceive the humanoid integrated system. In Figure 2 the main concepts in Figure 1 are shown with the successful prototypes that are used for experimental characterization like in ${ }^{7}$ and for a final design of the LARMbot humanoid robot with desired characteristics of an overall size of $70 \mathrm{~cm}$ tall and $5 \mathrm{~kg}$ weight. In Figure $2 \mathrm{~A}$ the torso prototype is shown as completed with sensing head and powered arms with a capacity of a payload of more than twice its own weight. The new developed leg systems prototype is shown in (Figure 2B) as the biped locomotion

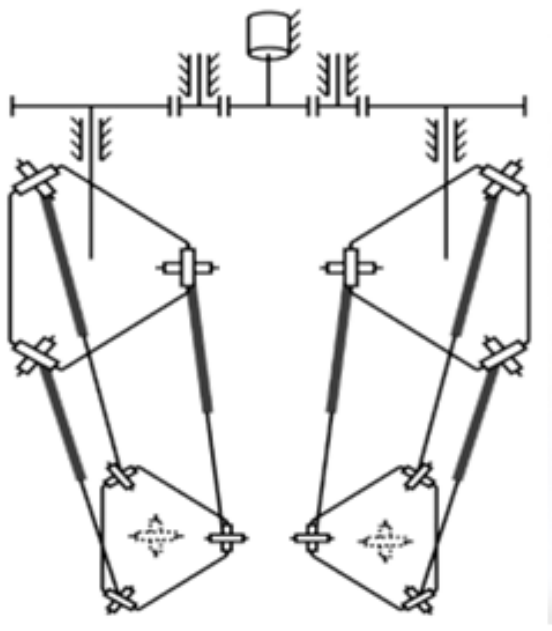

B

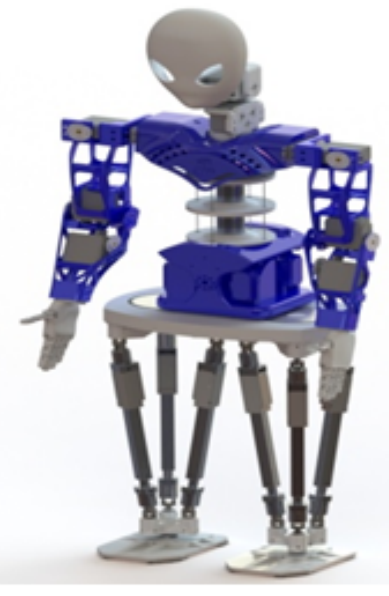

$\mathrm{C}$

system with the two leg tripods with linear actuators supporting the torso and its payload. The first assembly of the LARMbot Humanoid is shown in Figure $2 \mathrm{C}$ as a successful integration of the systems that have been developed independently and for integration on such a final design. The integration of structures and their performance has required and still require adjustments both in size and operation capabilities of the components for an optimized version of the humanoid that can be later equipped with more sensors and capabilities as depending of the application fields that are planned in servicing humans in home life or in job special tasks such as assisting elderly people, working in Cultural Heritage frames or in Industrial risky environments. 


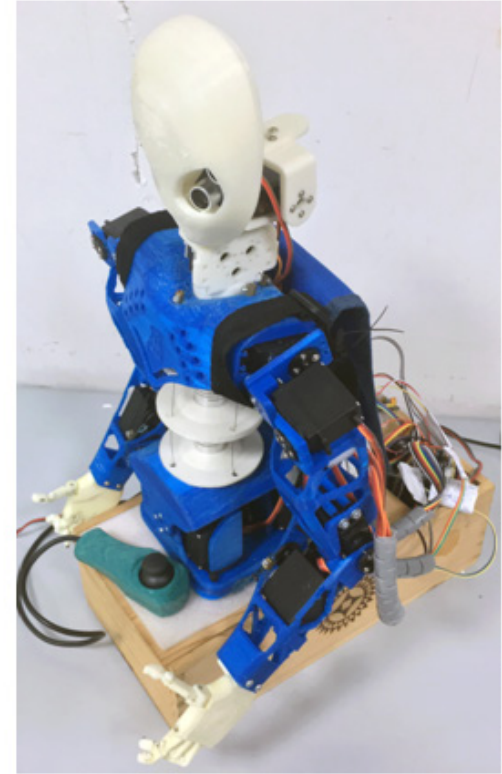

A

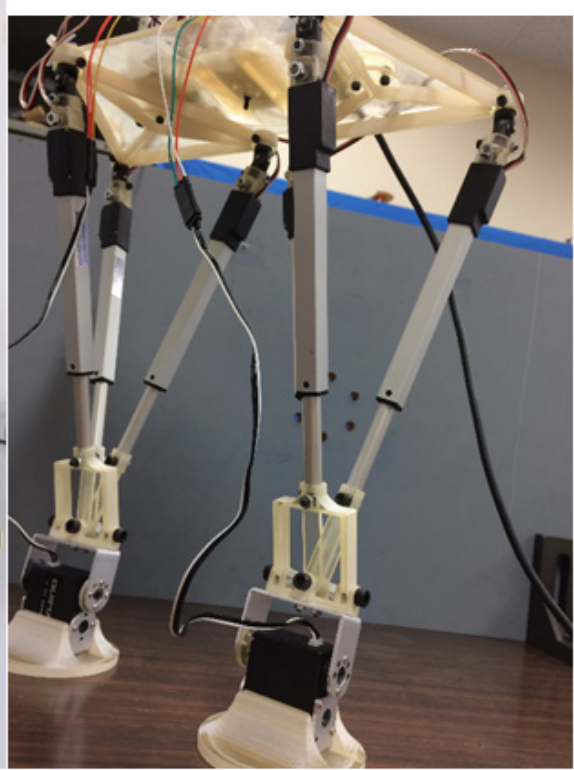

B

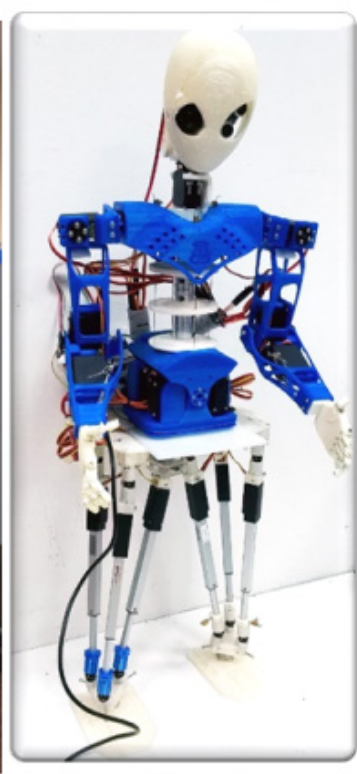

$\mathrm{C}$

Figure 2 Prototypes of the LARMbot Humanoid. (A) Torso; (B) Biped locomotor; (C) A firstfull assembly.

\section{Conclusion}

The LARMbot humanoid robot is designed as characterized by structures with parallel manipulator architectures and their mechanism design with low-cost features and user-oriented operation. The main focus of the current design goals is on mechanical design and its motion and payload capability, whereas complementary multisensorial and intelligent capabilities are still to be properly attached.

\section{Acknowledgements}

None.

\section{Conflict of interest}

Author declares that there is no conflict of interest.

\section{References}

1. Ceccarelli M. An Illustrated History of LARM in Cassino, Proceedings of RAAD 2012 international Workshop on Robotics in Ale-Adria-Danube Region, Napoli. Edizioni Scientifiche e Artistiche. 2012:35-42.
2. Ceccarelli M. Kinematic design problems for low-cost easy-operation humanoid robots. Interdisciplinary Applications of Kinematics. 2014;26:91-99.

3. Liang C, Ceccarelli M. Design and simulation of a waist-trunk system for a humanoid robot. Mech Mach Theory. 2012;53:50-65.

4. Cafolla D, Ceccarelli M. Design and Simulation of a Cable-Driven Vertebra-Based Humanoid Torso. International Journal of Humanoid Robotics. 2016;13(4):1-27.

5. Russo M, Ceccarelli M. Kinematic Design of a Tripod Parallel Mechanism for Robotic Legs. Mechanisms Transmissions and Applications. 2018;52:121-130.

6. Cafolla D, Wang M, Carbone G, et al. LARMbot: A New Humanoid Robot with Parallel Mechanisms. ROMANSY 21 - Robot Design, Dynamics and Control. 2016;569:275-283.

7. Cafolla D, Ceccarelli M. An experimental validation of a novel humanoid torso. Journal Robotics and Autonomous Systems. 2017;91:299-313. 